hypoglycaemia in neonates receiving PN 2012, and the UK National Confidential Enquiry into Outcome and Death (NCEPOD) recommendations 2010 for venous access.

Methods A questionnaire was devised by a pharmacist, paediatrician and neonatologist. Questions focused on key areas commonly encountered in routine PN practise, for which guidance is available. These included protein and lipid introduction, monitoring and complications of lipids, management of hyperglycaemia and venous access. One researcher conducted a telephone survey to registrars working in all 58 level 3 neonatal intensive care units (NICU) throughout the UK.

Results The response rate was 58/58 units (100\%). For preterm neonates requiring $\mathrm{PN}$, protein is commenced on day 1 in $88 \%$ of units and lipids by day 3 in $91 \%$. Most units exclusively use central lines for $\mathrm{PN}$ administration. All units use $\mathrm{x}$-ray verification of catheter tip position with 19 units also using contrast. Triglyceride levels are not monitored in 22 units. Management of hyperglycaemia is variable, with 25 units using insulin first line and not altering glucose infusion. Seven units avoid insulin use completely.

Conclusion Many nutritional support practises were consistent and in line with guidelines. However over a third of units fail to monitor triglyceride levels despite the known consequences of high lipid infusions and recommendations for monitoring. The high usage of insulin in the management of hyperglycaemia may not be advantageous considering recent findings around the risks of hypoglycaemia and mortality. The use of contrast for line verification is not nationally standardised.

\section{G59(P) LONG-TERM PSYCHOLOGICAL AND SOCIAL IMPACT REPORTED BY CHILDHOOD CRITICAL ILLNESS SURVIVORS: A SYSTEMATIC REVIEW AND THEMATIC SYNTHESIS}

doi:10.1136/archdischild-2013-304107.071

JC Manning, P Hemingway, S Redsell. School of Nursing, Midwifery and Physiotherapy University of Nottingham, Nottingham, UK

Aims Over 250,000 children annually in the US and UK experience critical illnesses. Advances in medicine and care have significantly reduced mortality, with over $95 \%$ of patients that survive. However, the long term psychological and social impact, as reported by children and adolescents that survive critical illness, is unclear. This paper reports a systematic review and thematic synthesis of qualitative literature that aims to explore and understand these phenomena.

Methods CINAHL; MEDLINE; PMC; PsychINFO; ASSIA and Dissertation and Thesis Databases were searched between 1806 and February 2012. Studies were selected using eight predetermined criteria (e.g. only qualitative empirical studies) and methodological quality was assessed using two standardised checklists $(1,2)$. Thematic synthesis (3) was used to extract, code and synthesise data.

Results From the 1307 studies identified, three met the inclusion criteria which were all of moderate methodological quality. All included studies were conducted in the UK involving a total of 51 participants aged 1-25 years. Reasons for participant critical illness were predominantly due to head/spinal injury, sepsis, or malignancies.

Eighty-six codes and six novel themes emerged from the data. Themes included: confusion and uncertainty; other people's narratives; focus on former self and normality; social isolation and loss of identity; transition and transformation. Analytical synthesis culminated in a framework which attempts to conceptualise the childhood survivors' journey.

Conclusions Childhood critical illness can expose survivors to a complex trajectory of recovery with residual psychosocial adversity manifesting in the long-term. Health professionals need to comprehend and support the potential complex psychological and social needs that may occur. Further empirical research is required to explore and test the conceptual framework developed from this thematic synthesis.

\section{REFERENCES}

1. Walsh D, Downe S. Appraising the quality of qualitative research. Midwifery. 2006; 22:108-19.

2. Thomas J, Sutcliffe K, Harden A, et al. Children and Healthy Eating: A systematic review of barriers and facilitators. London: EPPI-Centre, University of London 2003.

3. Thomas J, Harden A, Oakley A, et al. Integrating qualitative research with trials in systematic reviews. BMJ. 2004; 328:1010-2.

\section{G60(P) THREE YEAR RETROSPECTIVE REVIEW OF VIRAL RESPIRATORY INFECTION IN PAEDIATRIC INTENSIVE CARE ADMISSIONS}

doi:10.1136/archdischild-2013-304107.072

${ }^{1} \mathrm{O}$ Omolokun, ${ }^{2} \mathrm{C}$ Moore, ${ }^{1} \mathrm{M}$ Jardine. 'Paediatric Intensive Care, University Hospital of Wales, Cardiff, UK; ${ }^{2}$ Public Health Wales, UK

Introduction During the H1N1 2009 Pandemic season, all children ventilated on Paediatric Intensive Care (PICU) for respiratory failure had respiratory samples taken for virology screen.

H1N1 (2009) was declared eradicated both national and internationally in August 2010. We reviewed results of samples taken during and beyond this period and compared the respiratory isolates.

Methods All Nasopharyngeal Aspirates, Non -direct bronchoalveolar lavage or tracheal secretions from admissions in the months of September to April of 2009/2010, 2010/2011 and 2011/2012 were reviewed. The months of May to August of each year were regarded as off peak and so left out of the study. PICU admissions, discharges and death notifications records were correlated with virology reports.

Results Samples were processed from 287 PICU patients or episodes. $192(67.1 \%)$ of the children were ages $0-2$ years. (154)53.8\% of the patients were male. One or more Respiratory viruses was isolated in 159 samples (55\%). RSV accounted for $77(48.4 \%)$ of the positive samples, Rhinovirus 45(28.6\%), Para-influenza virus Type14, 14(8.8\%), Adenovirus 9(5.7\%), Pandemic H1N1 (2009) 8(5.1\%), Influenza A or B $3(1.9 \%)$ and Human Metapneumovirus $2(1.3 \%)$. Coinfection was found in $12(7.5 \%)$ of the positive samples.

Oseltamivir (Tamifu) was prescribed to positive cases in the population. Resistance to Oseltamivir was reported in one case treated. There were ten $(3.4 \%)$ mortalities from the study population two of whom were positive for Pandemic H1N1 (2009). A serious co-morbidity was present in all $10(100 \%)$ mortalities. We isolated a virus in $7 / 10(70.0 \%)$ deaths. No positive swabs for H1N1 (2009) was found after January 2011

Conclusion While we continue to carry out surveillance for sporadic or seasonal cases of H1N1 (2009), with the pandemic truly over, resources needs to be devoted to common respiratory viruses with greater burden of disease.

\section{G61(P) SEVERE DIABETIC KETOACIDOSIS: THE BENEFITS OF 'PHONING A FRIEND'}

doi:10.1136/archdischild-2013-304107.073

${ }^{1} \mathrm{~K}$ Bakaya, ${ }^{2 \mathrm{~K}}$ Parkins, ${ }^{1,2} \mathrm{R}$ Barber, ${ }^{2,3} \mathrm{R}$ Phatak, ${ }^{2} \mathrm{~S}$ Santo. ${ }^{1}$ PICU, Central Manchester University Hospitals NHS Foundation Trust, Manchester, UK; ${ }^{2}$ North West and North Wales Paediatric Transport Service, Regional Retrieval Services, Manchester, UK; ${ }^{3} P I C U$, Alder Hey Children's NHS Foundation Trust, Liverpool, UK

Aim To evaluate the management and outcome of children with severe DKA who were referred by different District General hospitals within the region to Regional retrieval team for advice.

Method Retrospective audit of all patients with diagnosis of DKA, referred to Regional Retrieval Team between November 2010 - June 